Revue scientifique sur la conception et l'aménagement de l'espace

\title{
Premières neiges : le paysage d'hiver dans les enluminures
}

First snow: the winter landscape winter landscape in illuminations (XIV th $-X V I^{\text {th }}$ centuries)

\section{Alexis Metzger}

\section{(2) OpenEdition}

\section{Journals}

Édition électronique

URL : http://journals.openedition.org/paysage/15796

DOI : $10.4000 /$ paysage. 15796

ISSN : 1969-6124

Éditeur :

École nationale supérieure du paysage de Versailles-Marseille, Institut national des sciences appliquées Centre Val de Loire - École de la nature et du paysage, École nationale supérieure d'architecture et de paysage de Bordeaux, École nationale supérieure d'architecture et de paysage de Lille, Agrocampus Angers

\section{Référence électronique}

Alexis Metzger, «Premières neiges : le paysage d'hiver dans les enluminures », Projets de paysage [En ligne], 8 | 2012, mis en ligne le 06 octobre 2012, consulté le 11 mars 2021. URL : http:// journals.openedition.org/paysage/15796 ; DOI : https://doi.org/10.4000/paysage.15796

Ce document a été généré automatiquement le 11 mars 2021.

Projets de paysage 


\title{
Premières neiges : le paysage d'hiver dans les enluminures
}

\author{
First snow: the winter landscape winter landscape in illuminations $\left(X V^{\text {th }}-X V I^{\text {th }}\right.$ \\ centuries)
}

Alexis Metzger

\section{Introduction}

1 Si les Grecs et les Latins avaient une vision des saisons, vision paysagère (Baridon, 2006) ou poétique (Dehon, 2002), l'hiver et la neige ont été redécouverts à la fin du Moyen Âge et à la Renaissance. Cette "morte-saison » va occuper une place de plus en plus importante dans les arts au point de devenir peu à peu apprivoisée, voire célébrée.

2 Cette « renaissance » de l'hiver s'inscrit à un moment charnière de l'Histoire. C'est en effet entre les $\mathrm{XIV}^{\mathrm{e}}$ et $\mathrm{XV}^{\mathrm{e}}$ siècles qu'un ensemble de faits, représentations, invention, etc., forment une transition entre le Moyen Âge et la Renaissance. Cette transition, économique, sociale et culturelle, est aussi climatique : après une période assez chaude, le petit optimum médiéval, l'Europe va connaitre une série d'hivers froids et d'étés frais : le petit âge glaciaire (vers 1300-1850 selon Emmanuel Le Roy Ladurie, 2004).

3 La représentation de la saison hivernale s'est construite dans les enluminures des livres d'heures de plus en plus répandus à la fin du Moyen Âge. Elles ouvrent une fenêtre sur le paysage et sont les premières représentations du temps météorologique et du temps qui passe. Ce qui nous intéresse dans cet article est double : voir comment se construit le paysage hivernal et quelles caractéristiques de l'hiver ont été préférées dans les enluminures. Les artistes ont-ils mis l'accent sur tel ou tel phénomène météorologique? Alors que l'hiver est une saison marquée par la difficulté des conditions de vie pour une majorité d'hommes, est-ce cette réalité qui transparaît dans les enluminures?

4 C'est donc le rapport entre paysage et représentations qui sera ici interrogé, en mettant en balance ce qu'on sait du paysage de l'époque et les représentations qui lui sont liées. 
Car si un paysage n'existe qu'à partir du moment où l'on veut bien lui prêter attention, le reconnaître comme tel, rien ne dit que les représentations que les artistes en font vont être en symbiose avec ce regard. En d'autres termes, le support qu'est la miniature présente un morceau choisi non pas d'une nature qui existerait en soi, mais d'un regard qui se porte sur elle. D'où différentes strates de lecture-regard du paysage à déchiffrer dans une miniature : celle, en premier lieu, du simple regard qui fait paysage, et celle, ensuite, du regard de l'artiste qui fixe sur une miniature ce regard (voir notamment Berque, 2000 et Luginbühl, 1996).

Grâce au groupe d'archéologie médiévale de l'EHESS, nous avons constitué un corpus qui rassemble la plupart des images hivernales produites du XIV au XVI ${ }^{e}$ siècle (jusque vers 1510-1520). Plusieurs bases de données ont été consultées pour dresser ce panorama le plus exhaustif possible des représentations hivernales. Excepté quelques fresques que nous présenterons rapidement, l'analyse portera exclusivement sur les miniatures hivernales dans les livres d'heures. Ce sont les seules images de l'hiver qui existent pour cette période, la peinture de paysage d'hiver étant bien plus tardive (Metzger, 2012) ${ }^{1}$. Ces livres sont élaborés dans des ateliers d'enlumineurs laïcs qui se multiplient dès la fin du xiII ${ }^{\mathrm{e}}$ siècle dans les Flandres.

Dans un premier temps, nous verrons en quoi le nouveau regard qui se tourne vers le temps qu'il fait au Moyen Âge a été déterminant dans la découverte du paysage. Les éléments climatiques ou météorologiques jouent le rôle d'« effets de réel » qui ancrent les travaux des champs dans leur environnement. Puis, nous présenterons une chronologie des premières représentations de l'hiver afin de dégager les grandes étapes de la construction du paysage d'hiver. Enfin, nous montrerons quelle imagerie de l'hiver a dominé dans les enluminures en proposant des éléments d'interprétation pour l'expliquer.

\section{Mais où sont les neiges d'antan?}

7 Retracer les processus qui ont conduit à faire du paysage un genre indépendant est extrêmement complexe. Plusieurs auteurs ont souligné à quel point diverses influences s'exerçaient, à différentes époques et dans différents lieux. «Il s'agit d'un processus dans lequel toutes les formes d'imagination étaient convoquées et qui concerne l'entière attitude de l'homme envers son environnement physique.» (Pearsall, 1973.) Cette évolution correspond à un changement dans les représentations mentales des hommes et femmes du Moyen Âge.

\section{L'hiver, une morte-saison?}

Quelques traces poétiques ou historiques permettent d'appréhender ce qu'était l'hiver au Moyen Âge et de lui donner un semblant de définition temporelle et sociale. L'expression « morte-saison » résume sans doute le mieux cette définition (La Soudière, 1987). Elle apparaît au vers 10 du Lais de François Villon: «En ce temps que j'ay dit devant,/Sur le Noël, morte saison,/Que les loups (se) vivent du vent/Et qu'on se tient en sa maison/Pour le frimas, près du tyson. » Cette évocation poétique de l'hiver traverse les siècles. Elle est accompagnée de conditions climatiques spécifiques : le froid, le gel et la neige contre lesquels les individus pouvaient difficilement se prémunir. Selon les historiens, l'hiver est la saison des contraintes : la faim taraude souvent le paysan et la 
mort est redoutée. Le froid est souvent synonyme de disette, voire de famine et de surmortalité, ce à quoi il faut ajouter la grande peste qui emporte Jeanne de Valois, reine de France, en 1349. "Même clément, ajoute Georges Duby, l'hiver signifiait pour eux (les pauvres) l'embauche difficile, les journées courtes et les salaires bas; puis les rémunérations s'élevaient avec le soleil... » (1993).

Dans les poésies, l'hiver commence à la Toussaint et finit à Pâques, comme on le lit dans la balade 958 de Eugène Deschamps : «Prince, mettez sur ce fait atrempance :/De la Toussains, que li hyvers commence/Jusqu'a Pasques, nous le vous supplions. » Ces vers rappellent, là encore, la grande peur de l'hiver. Eugène Deschamps naît d'ailleurs en 1300 , presque avec les premières années de grand froid après une période d'« optimum médiéval» (vers 1000-vers 1300) plutôt douce (Alexandre, 1976 et 1987). Les bornes temporelles de la saison hivernale mentionnées par le poète semblent coller à la réalité vécue par les sociétés du Moyen Âge. Comme le résume Fleur Vigneron (2002): "L'hiver va de novembre à mars parce qu'il fait mauvais et que les travaux des champs sont réduits. » Aux Pays-Bas, c'est la St-Maarten, le 11 novembre, qui symbolise chez les paysans l'entrée dans l'hiver (Van Straaten, 1977).

\section{Un « effet de réel » climatique}

$10 \mathrm{Au}$ temps des premières représentations picturales, le paysage occupe une place marginale. Les rares éléments naturels offerts au spectateur sont relégués au second plan et l'accent est mis plutôt sur les hommes et leurs activités. Mais au fil du temps, ces activités (religieuses, agricoles...) vont peu à peu s'ancrer dans leur environnement, et les traces de la nature se font de plus en plus présentes. Car si le chrétien entend le message se dévoilant à travers les représentations, il apparaît que ce message sera beaucoup mieux intégré si le quotidien est présent, bref si ce message passe par l'image : «Elle (l'image) offre la plus efficace entremise entre la parole de Dieu et la mimique fervente par quoi le corps et l'âme s'arrachent à tout ce qui les attache " (Duby, 1993). En somme, inventer le paysage répond sans doute beaucoup plus à un souci moral qu'esthétique. Il s'agit de créer un "effet de réel» qui fait prendre conscience aux laïcs que la spiritualité s'exerce au quotidien. Quel plus bel effet alors que d'encadrer certaines scènes bibliques d'éléments naturels? Ces arbres, collines, champs... ancrent la parole du Christ dans la réalité.

11 Or, la réalité pour les hommes et femmes du Moyen Âge est dictée en grande partie par le climat. Les sources historiques le rappellent bien : Tel été " pourri » pourra entraîner disettes et famines, tel hiver rude nombre de décès (voir notamment Alexandre, 1976 et 1987 et Le Goff, 1997). En ce sens, ancrer le message biblique dans la réalité vécue, c'est aussi l'ancrer dans la réalité climatique de l'époque. D'ailleurs, représenter le temps qu'il fait et le temps qui passe ajoute non seulement un nouvel effet de réel, les cycles des saisons étant liés aux cycles chrétiens, mais renforce la place des éléments atmosphériques dans les représentations. C'est ainsi que vont s'esquisser du début du $\mathrm{XIV}^{\mathrm{e}}$ siècle à la fin du $\mathrm{XV}^{\mathrm{e}}$ siècle des paysages climatiques de plus en plus fréquents et de plus en plus fidèles à la réalité. Même si le symbolisme religieux est fort, ces représentations sont bel et bien des représentations du temps météorologique. 


\section{La naissance du paysage d'hiver}

\section{dans les paysages : la glace et la neige. Dans un grand nombre de représentations des} origines, ils n'apparaissent pas. Cela traduit peut-être une peur d'exposer son corps à l'extérieur quand les moyens pour se protéger du froid étaient dérisoires (cf. supra). Nombre de miniatures font d'un homme se réchauffant près du foyer le symbole de l'hiver et, a fortiori, ne montrent pas de neige, à tel point que Derek Pearsall parle d'un "February-by-the-fire motif» (Pearsall, 1973).

13

tre image traditionnellement liée à l'hiver est le repas, souvent celui de Noël. La richesse de certains commanditaires de calendriers va de pair avec le faste des banquets représentés, principalement au mois de décembre. Pour les personnes plus pauvres, une scène intimiste est préférée. Parfois, un couple dîne seul à la chaleur d'un feu. Là encore, ces scènes intérieures ne donnent aucune place à la représentation des phénomènes atmosphériques. Sans doute permettent-elles d'entretenir une illusion et de «masquer la triste réalité du monde hors des murailles du château » (Henish, 1999).

Seules les miniatures associant des vues de l'intérieur d'une maison et de l'extérieur vont montrer la neige. Ce regard tourné vers l'extérieur et la nature, émergeant au Moyen Âge, participe indéniablement de la naissance du paysage. Il va progressivement s'imposer dans les miniatures et transformer l'image des hivers: "C'est seulement à partir du Xve siècle que les enluminures mettent en scène la neige et le froid » (Mane, 2004). Quelques images font cependant figure d'exception auparavant.

\section{Les images liminaires de l'hiver}

Retrouver les premières traces de neige dans les représentations n'est pas simple. La première chute de neige est sans doute l'œuvre de Barthélemy l'Anglais dans Le Livre des propriétés des choses écrit vers 1240 (une version en langue provençale de ce livre, datée vers 1350, est conservée à la bibliothèque Sainte-Geneviève, Paris, 1029 132v, voir Alexandre-Bidon, 1993). Mais la miniature est de toute petite taille. Ambrogio Lorenzetti va quant à lui représenter une chute de neige sur la fresque du Palazzo Pubblico de Sienne. Un personnage symbolisant l'hiver, vieux et bien emmitouflé, apparaît en effet dans un médaillon situé au-dessus de la fresque du Mauvais gouvernement. Cette symbolique de l'hiver va être permanente au cours des siècles et nombre de représentations, dont les gravures, personnalisent l'hiver sous les traits d'un tel homme. Sur la fresque de Lorenzetti, nulle trace d'un quelconque plaisir. Même si l'homme tient une boule de neige, il semble avoir une vie rude. La neige, tombant dru, renvoie une image négative de l'hiver.

Mais c'est dans un manuel lombard de la seconde moitié du XIve siècle que la neige refait véritablement son apparition (Tacuinum Sanitatis, BNF, nouv. acq. lat. 1673, fol. $96 \mathrm{v}$, figure $\mathrm{n}^{\circ} 1$, voir image en cliquant ici : http://blog.bnf.fr/uploads/lecteurs/ 2010/12/nal1673.jpg). L'image y est positive : deux adultes, plutôt aisés, aux vêtements légers et peu adaptés au froid, se lancent des boules de neige au premier plan alors que des montagnes composent le second. À noter que l'imaginaire de la neige semble lié à celui des montagnes. Même si un manteau neigeux disséminé recouvre le sol du premier plan, sur les bords d'un lac gelé, des cimes enneigées évoquent peut-être le froid et la rudesse des conditions de vie. Le paysage est irréaliste. 

illustrés, ils font état, par l'image, de tout ce qui pouvait influencer la santé (plantes, aliments, vie quotidienne, saisons...). Des paysages extérieurs servent rarement d'illustration à l'hiver, car la médecine préconisait «feu et vêtements » pour bien passer l'hiver (Poirion et Thomasset, 1995). Il faut en effet rapprocher ces choix picturaux à la théorie des humeurs qui, bien après Hippocrate, connaît un grand succès au Moyen Âge. À l'hiver est associé un élément, l'eau, une humeur, le flegme, et un tempérament, «flegmatique ».

Peinte vers 1400, une fresque marque la naissance du paysage enneigé en Europe occidentale. Située à Trente, au château de Buonconsiglio, elle s'inscrit dans la tradition de l'art lombard, profane, et semble inspirée par la fresque de Lorenzetti. Elle dépeint les mois de l'année. En décembre, des paysans coupent du bois dans la montagne et le ramènent dans une ville fortifiée. Le manteau neigeux est peu épais et la neige recouvre le sol mais pas les arbres. En janvier, le manteau neigeux est bien plus important (voir image en cliquant ici: http://www.photo.rmn.fr/cf/htm/CSearchZ.aspx? $\mathrm{o}=\&$ Total $=8 \& \mathrm{FP}=73306777 \& \mathrm{E}=2 \mathrm{~K} 1 \mathrm{KTSGW} 2 \mathrm{FOAP} \& S I D=2 \mathrm{~K} 1 \mathrm{KTSGW} 2 \mathrm{FOAP} \& N e w=T \& P i c=7 \& S u b E=2 \mathrm{C} 6 \mathrm{NU} 0 J U Z 61 Q$ ). l'extérieur des murailles du château. D'autres partent à la chasse, aidés d'un bâton. Un vrai témoignage de la vie en hiver nous est donné à voir. Même si les proportions sont encore irréalistes - les deux groupes se lançant des boules de neige étant situés à environ un mètre de distance par exemple -, ces deux fresques sont novatrices dans la représentation de la neige.

On assiste donc ici aux balbutiements des représentations neigeuses. Les auteurs des peintures commencent à prendre en compte les phénomènes atmosphériques et la nature en général. Comme le dit Umberto Eco, c'est l'époque d'« un Moyen Âge tardif où frémissent déjà de nombreux pressentiments de la Renaissance » (dans Cazelles, 1988). Liée à des scènes profanes ou religieuses, la neige fait à la fin du $\mathrm{XIV}^{\mathrm{e}}$ siècle son apparition dans l'iconographie médiévale. Ce météore va être repris dans plusieurs miniatures $\mathrm{du} \mathrm{Xv}^{\mathrm{e}}$ siècle. Reprenant les temps liturgiques et astronomiques, ces calendriers vont connaître un foisonnement aux $\mathrm{Xv}^{\mathrm{e}}$ et $\mathrm{XVI}^{\mathrm{e}}$ siècles en Europe. Plusieurs enlumineurs célèbres se partageront la tâche de les illustrer dans la mesure où le commanditaire est assez riche pour s'offrir leurs services.

21 Ainsi, à la fin du XIV siècle, Jacquemart de Hesdin réalise pour le duc de Berry les enluminures des Petites Heures du duc de Berry et va représenter la première nativité « dans » la neige. Réalisée avant 1388, elle montre Jésus naissant dans une ferme et, à l'extérieur, un manteau neigeux épais. C'est la première fois que le blanc de la neige occupe une telle place dans une enluminure. Puis, au début du $\mathrm{xv}^{\mathrm{e}}$ siècle, Christine de Pisan, auteur de la Cité des dames, fait illustrer par Jacquemart de Hesdin un livre d'heures atypique mettant en scène les grandes femmes de l'Histoire. Deux enluminures illustrent des événements se déroulant en présence de neige ${ }^{2}$.

Selon Millard Meiss, il est fort probable que Paul de Limbourg se soit inspiré de ces deux miniatures pour proposer sa vision du mois de février dans les Très Riches Heures $d u$ duc de Berry, sans doute la plus célèbre de toutes les illustrations de ce mois dans les calendriers des livres d'heures de l'histoire médiévale (Meiss, 1974). 


\section{Février, dans les Très Riches Heures du duc de Berry}

Très Riches Heures, écrit Umeberto Eco, sont un documentaire cl une machine visuelle qui nous raconte la vie d'une époque "(dans Cazelles, 1988). Commandé par le fils du roi Jean le Bon, elles font suite à d'autres livres déjà somptueux. Le duc de Berry s'entoure en effet des plus talentueux enlumineurs du début du $\mathrm{XV}^{\mathrm{e}}$ siècle : Jacquemart de Hesdin décore en 1409 les Grandes Heures, les frères de Limbourg les Belles heures. C'est à partir de 1410 que ces derniers commencent à décorer les Très Riches Heures. Leur travail s'interrompt à la mort du duc en 1416. Parmi les illustrations de ce livre d'heures, certaines sont les premières à donner à voir le paysage, à commencer par celles du calendrier. caractéristique du Trecento. Tel est le cas pour le mois de février (voir image en cliquant ici ou là. Mettre le lien http://commons.wikimedia.org/wiki/ File:Les_Tr\%C3\%A8s_Riches_Heures_du_duc_de_Berry_f\%C3\%A9vrier.jpg).

D'ailleurs, Paul de Limbourg, son auteur selon Millard Meiss qui a vu plusieurs caractéristiques communes entre son œuvre et cette miniature, avait fait un voyage en Italie. Il a probablement visité Sienne et son Palazzo Pubblico. La fresque de Lorenzetti lui a-t-elle servi de modèle ? Est-il allé jusqu'à Trente et a-t-il vu la fresque de la chapelle du Torre dell'Aquila? Au-delà des discussions entre historiens d'art, il est certain que les influences du calendrier des frères de Limbourg sont nombreuses, et peut-être italiennes.

Malgré ces emprunts et inspirations possibles, la miniature du mois de février est novatrice à plus d'un titre. C'est la première fois tout d'abord que sont donnés à voir autant de détails en hiver : la cour de ferme au premier plan, l'homme revenant avec du bois, le village sous la neige dans le lointain... Autant de motifs parfois déjà représentés mais jamais avec autant de finesse et surtout jamais rassemblés dans un paysage. Il ne s'agit plus cette fois-ci d'illustrer l'hiver grâce à un unique symbole (le vieil homme se réchauffant) ou une activité (les boules de neige) mais de montrer comment est vécu l'hiver enneigé dans une campagne quelconque d'Europe. Évidemment, il s'agit encore d'un paysage symbolique. Il procède par assemblage de motifs séparés et ne constitue pas une vue faite d'après nature: «Les détails s'ordonnent dans une intention décorative » (Clark, 2006.)

S'opère ensuite une inversion des points de vue. Dans la Nativité des Petites Heures, l'extérieur enneigé était vu de l'intérieur. Ici, c'est l'inverse : le lecteur-spectateur de cette scène campagnarde est placé à l'extérieur. Il voit un paysage enneigé et l'intérieur d'une ferme grâce à l'absence fictive d'un mur. Il y a enfin dans cette œuvre une maîtrise des couleurs de l'hiver jamais atteinte. "La richesse de cette évocation de l'hiver tient aussi à l'heureuse intervention de la couleur. Limitée sans doute, mais si efficacement distribuée "(Collot, 1992). Vécue au quotidien, la neige marque ici le temps du repos dans la ferme, mais aussi des difficultés pour se déplacer.

n dépit de leur éclatante nouveauté, les Très Riches Heures n'ont pas eu d'effet immédiat. "L'esprit des Limbourg " (Meiss, 1974) semble mettre presque un siècle à se diffuser. En effet, le paysage de neige n'intéresse guère les artistes au cours du $\mathrm{XV}^{\mathrm{e}}$ siècle : «Il est frappant de remarquer que les miniaturistes attendent le tout début $\mathrm{du} \mathrm{XVI}{ }^{\mathrm{e}}$ siècle pour l'illustrer couramment (la neige) [...]. Jusqu'alors, l'hiver neigeux 
était synonyme de corvées, de lutte contre les animaux sauvages, et les scènes intimes lui étaient amplement préférées » (Alexandre-Bidon, 1993).

\section{Les caractéristiques du paysage hivernal}

Grâce à une nouvelle place donnée à la nature et à la maîtrise progressive de la perspective, les paysages d'hiver se construisent. L'espace est de plus en plus " homogénéisé », organisé en harmonie vraisemblable. Il semble pouvoir être embrassé d'un seul regard. Certes, les scènes d'intérieur plus traditionnelles des livres d'heures en hiver ne disparaissent pas pour autant. L'image du vieil homme se réchauffant est toujours bien présente, mais n'est plus omniprésente.

Si l'on s'intéresse aux miniatures hivernales qui ont été peintes jusque vers 1510-1520, on peut dégager les grandes caractéristiques des représentations. Nous avons choisi de les classer selon quatre grandes entrées qui composent le paysage hivernal : le temps météorologique, l'espace, les hommes et les activités humaines. Les tableaux sont volontairement synthétiques.

\section{Temps}

Tableau 1. Les différents types de temps d'hiver dans les miniatures

\begin{tabular}{|ll|}
\hline Temps metteorologlque représenté & Occurrence \\
\hline Glace & Assez fréquent \\
\hline Chute de neige & Assez fréquent \\
\hline Pluie & Rare \\
\hline Manteau neigeux & Très fréquent \\
\hline Arbres dépouillés de feuilles & Très fréquent \\
\hline Vent & Rare \\
\hline Clel dégagé & Fréquent \\
\hline Círus ou cirrocumulus & Fréquent \\
\hline Stratus ou stratocumulus & Rare \\
\hline Cumulus ou cumulonimbus & Rare \\
\hline
\end{tabular}

31 À partir du moment où les enlumineurs s'intéressent au paysage, ils vont représenter des hivers enneigés. Un manteau neigeux, de faible épaisseur, est une des caractéristiques récurrentes des miniatures hivernales. La neige n'est pas gelée, car des empreintes sont souvent figurées. Elle recouvre également les toits des maisons, des églises ou des branches d'arbres dépouillés de feuilles. Dans un certain nombre de miniatures, de la neige tombe. On peut distinguer des flocons de neige très fins ou des flocons plus denses en eau. Dans une des miniatures, l'intensité du froid n'est pas suffisante pour que la neige tienne au sol (Heures à l'usage de Rome, début $\mathrm{du} \mathrm{XVI}$ siècle, BN, lat 10555). Dans une autre miniature, illustrant la traversée des Alpes par Hannibal, c'est une véritable tempête de neige qui assaille l'armée (BN, FR 00366, 19v, fin du $\mathrm{XV}^{\mathrm{e}}$ siècle).

La neige avait d'ailleurs une symbolique religieuse. Certains psaumes le rappellent: «Lave-moi et je serai plus blanc que neige » dit le psaume 51, associant ainsi la neige à la pureté (peut-être donc l'innocence de l'enfance). Le psaume 147 (versets 16 à 18) témoigne lui de la toute-puissance de Dieu sur terre, maître des saisons, inspirateur des 
chutes de neige : «Il fait tomber la neige comme de la laine, et répand le givre comme de la cendre; il jette sa glace par morceaux. Qui peut résister devant son froid? Il envoie sa parole, et les fait fondre; il fait souffler son vent, et les eaux s'écoulent. » Quelle plus belle illustration de ce psaume que certaines enluminures hivernales où des pelotons de neige tombent abondamment? «Signes d'élection, signes de réprobation, manifestation de la puissance divine, les phénomènes météorologiques annoncent ou accompagnent les événements qui marqueront la vie des grands de ce monde comme celle du petit peuple» (Ducos, 1988). En ce sens, la neige en hiver marque bien la vie de la société médiévale : elle définit une saison, un cycle et protège les graines de blé dans la terre lorsqu'elle forme un manteau. D'ailleurs, «la vie de chacun n'était-elle pas composée de saisons, que venaient clore les neiges de l'hiver?» (Muchembled, 1978.)

Lorsque la neige ne tombe pas, les temps d'hiver représentés sont souvent froids et secs. Ce type de temps est bien marqué par un voile de cirrus qui apparaît dans un ciel souvent bleuté. Le froid de ces temps secs est aussi rappelé par des stalactites qui s'accrochent aux toits, comme dans le Bréviaire Grimani, et par la glace qui fige les cours d'eau et les mares. À notre connaissance, rares sont les miniatures à couvrir le ciel de cumulus ou de cumulonimbus.

\section{Espace}

Tableau $n^{\circ} 2$. L'espace des miniatures

\begin{tabular}{|ll|}
\hline Espace représentés & Occurrence \\
\hline Ville & Fréquent \\
\hline Village & Très fréquent \\
\hline Campagne & Fréquent (au second plan) \\
\hline Plaine & Fréquent \\
\hline Coline & Fréquent \\
\hline Montagne & Rare \\
\hline
\end{tabular}

34 L'attention portée au paysage se lit dans la topographie des miniatures qui devient plus fidèle à la réalité. Alors que des montagnes alpines pouvaient, dans les premières représentations, côtoyer des plaines, les paysages sont nettement plus réalistes dès le $\mathrm{XV}^{\mathrm{e}}$ siècle. Les proportions et distances sont parfois peu vraisemblables mais elles s'harmonisent peu à peu.

En majorité, les miniatures correspondent aux espaces dans lesquels elles sont peintes, c'est-à-dire les Flandres. La représentation de montagnes est rare, certainement parce que les grands ateliers d'enlumineurs étaient situés en plaine. On doit également relier cette absence de montagnes dans les enluminures à l'histoire et à la géographie culturelles de ces paysages. Les Alpes, par exemple, ne sont découvertes, voire " inventées », qu'au XVII ${ }^{\mathrm{e}}$ siècle (Reichler, 2002 et Joutard, 1986). Bien que sans grande déclivité, la topographie est présente : les collines permettent de représenter différents plans et de figurer des lignes d'horizon.

On peut également relever une légère préférence des enlumineurs pour les villages par rapport aux villes. Dans tous les cas, aucune enluminure ne représente un paysage sans constructions humaines (bâtiments, palissades...). C'est bel et bien l'homme qui est le sujet principal des miniatures, sa vie et ses activités. 
, les scènes extérieures et intérieures apparaissent de plus en plus fréquemment sur la même miniature. On distingue souvent l'intérieur d'une ferme par une fenêtre. Dans la lignée de Paul de Limbourg, Simon Bening (résidant à Bruges) montre, quant à lui, l'intérieur d'une ferme et l'extérieur sous un blanc manteau neigeux. Au mois de février, dans le Bréviaire Grimani, il est présenté quasiment la même scène que la miniature de février des Très Riches Heures du duc de Berry. Une double image se dégage à nouveau : la tranquillité devant la cheminée et la rudesse de l'hiver à l'extérieur. Simon Bening ajoute cependant une touche comique: un enfant, dans la ferme, urine à l'extérieur, ce qui fait fondre la neige.

\section{Présence humaine}

Tableau $n^{\circ} 3$. Les hommes représentés dans les miniatures

\begin{tabular}{|ll|}
\hline Présence humaine & Ocourrence \\
\hline Nombreux & Fréquent \\
\hline Peu nombreux & Rare \\
\hline Enfants & Fréquent \\
\hline Adultes & Fréquent \\
\hline Vieillards & Assez rare \\
\hline
\end{tabular}

Dans les paysages des miniatures s'activent plusieurs membres de la famille. Aucune miniature ne montre un hiver sans hommes, ce qui s'explique par leur fonction. Des enfants sont souvent représentés. Peut-être faut-il y voir le symbole de la renaissance à chaque nouvelle année? En suivant Bridget Ann Henish, on peut dire que janvier montre plutôt la petite enfance (moins de 6 ans), et février des enfants de 6 à 12 ans. Le vieil homme, symbolisant l'hiver, apparaît aussi parfois encore en février mais disparaît au printemps. Ici, les saisons du calendrier épouseraient le rythme de la vie, la naissance étant soit en hiver, soit au printemps. Globalement donc, les scènes d'hiver sont plutôt familiales. Les parents vaquent à leur occupation tandis que les enfants jouent dehors ou se réchauffent dans la ferme.

À noter qu'aucune enluminure ne montre des personnes très pauvres ou des mendiants. Les représentations de la société varient selon le destinataire du livre d'heures qui y verra bien souvent le reflet de sa condition sociale et de sa vie. Tel était le cas dans les enluminures des Très Grandes Heures du duc de Berry qui incluaient les châteaux du duc à l'arrière-plan.

\section{Activités}


Tableau $n^{\circ} 4$. Les activités représentées dans les miniatures

\begin{tabular}{|c|c|}
\hline Actlvites représentées & Occurrence: \\
\hline Tuer le cochon & Fréquent \\
\hline Lancer des boules de neige, jouer & Fréquent \\
\hline $\begin{array}{l}\text { Se réchauffer devant l'âtre } \\
\text { (scène incluant une vue de } \\
\text { l'extérieur) }\end{array}$ & Assez fréquent \\
\hline Ramasser le bois & Assez fréquent \\
\hline Dégager les rues & Rare \\
\hline Se promener & Rare \\
\hline
\end{tabular}

40 Les miniatures présentent quelques fois un hiver festif et un manteau neigeux bienvenu. Ainsi, les Heures de la duchesse de Bourgogne datant du milieu du $\mathrm{xv}^{\mathrm{e}}$ siècle montrent un mois de décembre où toute une société s'amuse au grand air (voir image en cliquant ici : http://www.photo.rmn.fr/cf/htm/CSearchZ.aspx? $\mathrm{o}=\&$ Total $=13 \& \mathrm{FP}=73311905 \& \mathrm{E}=2 \mathrm{~K} 1 \mathrm{KTSGW} 2 \mathrm{NCPX} \& \mathrm{SID}=2 \mathrm{~K} 1 \mathrm{KTSGW} 2 \mathrm{NCPX} \& \mathrm{New}=\mathrm{T} \& \mathrm{Pic}=8 \& S u b E=2 \mathrm{C} 6 \mathrm{NU} 0 \mathrm{GKD} 820$ ). Plusieurs enfants et adultes se lancent des boules de neige, d'autres préparent un bonhomme de neige ou roulent une énorme balle qui servira de jeu en équipe. Dans tous les cas, ces neiges joyeuses marquent une nouvelle rupture dans les représentations. Danièle Alexandre-Bidon, retraçant l'histoire ce ces jeux et sports d'hiver au Moyen Âge, se demande ainsi si les enfants n'étaient jusqu'alors pas représentés dans les enluminures parce qu'ils ne sortaient pas l'hiver « de crainte d'un refroidissement mortel» (Alexandre-Bidon, 1993). À notre connaissance, aucune miniature ne montre cependant de fête hivernale comme mardi gras.

41 Si des activités plus contraignantes apparaissent également çà et là dans les miniatures, elles sont bien moins fréquemment représentées. Il en va ainsi de la chasse au loup (Calendrier avec éphémérides, BN, FR 01872, 14v, 1539) et du dégagement de la neige amassée dans les rues (assez rare, voir le mois de février dans un livre d'Hheures de la fin $\mathrm{du} \mathrm{Xv}^{\mathrm{e}}$ siècle, conservé à Lyon, bibliothèque municipale, ms 514). L'abattage des arbres était selon les régions toléré pour effectuer quelques travaux de réparation (clôtures...) et/ou pour se chauffer. Mais aucune miniature ne montre de guerre ou de pillage de villages en hiver, pourtant fréquents.

Ce sont bien les activités « physiques » qui sont majoritaires. L'hiver est loin d'être oisif dans les miniatures qui représentent un paysage. L'humeur "flegmatique", l'hibernation pourrait-on dire, s'éclipse au profit du travail. Couper le bois, porter des brindilles, tuer le cochon... autant de motifs qui apparaissent aussi bien lorsque de la neige tombe que lorsque le temps est sec. Certaines tâches semblent être attribuées couramment aux femmes : porter le bois ou recueillir le sang du cochon (Henish, 1999).

Comme le résume Bridget Ann Henish, les miniatures des hivers créent donc une imagerie éloignée de la réalité hivernale du petit âge glaciaire. Lié aux famines, à la maladie et au froid, l'hiver était " passionnément détesté au Moyen Âge ». Ces réalités transparaissent très peu dans les miniatures. Elles sont mises de côté au profit d'une «illusion» qui permettait à l'enlumineur de réaliser un calendrier harmonieux en gommant guerres et mortalité. Dans cette illusion disparaissaient également les malheurs liés au mauvais temps. Cette idéalisation du temps est ainsi le pendant de la société idéalisée : les représentations ne reflètent globalement pas l'hiver contraignant pour les pauvres, décrit par les sources écrites comme mortifère pour les animaux et les humains les plus faibles. 


\title{
Un paysage modèle
}

\begin{abstract}
en reprenant les tableaux construits précédemment $\left(\right.$ tableau $\left.^{\circ}{ }^{\circ}\right)$ : celle de paysages d'hiver, dans des campagnes enneigées planes ou modestement ondulées en collines où s'activent dehors les membres d'une famille.
\end{abstract}

Tableau $n^{\circ} 5$. Le paysage « modèle » de l'hiver aux XV et XVI siècles

\begin{tabular}{|c|c|c|c|c|}
\hline Lieu & Topographie & $\begin{array}{l}\text { Phénomène } \\
\text { météo }\end{array}$ & Personnages & Activités \\
\hline Campagne & Colline & $\begin{array}{l}\text { Manteaux } \\
\text { neigeux }\end{array}$ & Famille & $\begin{array}{l}\text { «Physiques } \\
\text { » }\end{array}$ \\
\hline
\end{tabular}

\section{Conclusion}

Les enluminures hivernales montrent comment les sociétés se représentaient leurs hivers. Elles permettent de réaliser comment le paysage hivernal devient un "vrai » paysage climatique. Largement irréaliste aux origines, le paysage d'hiver représenté s'harmonise peu à peu jusqu'à pouvoir être embrassé d'un seul regard dans les enluminures les plus abouties de Simon Bening par exemple, au début du XVI $\mathrm{e}^{\mathrm{e}}$ siècle.

Plusieurs facteurs religieux, économiques, culturels, voire climatiques (le petit âge glaciaire), sont intervenus dans cette construction du paysage d'hiver. Ils peuvent expliquer pourquoi une imagerie de l'hiver s'est créée. L'imagerie est positive et contraste avec les descriptions de l'hiver que nous donnent les historiens ou les poètes contemporains. Plutôt que de le subir, les hommes s'adaptent à l'hiver dans les enluminures, voire commencent à le maîtriser. C'est donc bel et bien une nouvelle vision de la nature qui se construit au fur et à mesure et dont les enluminures hivernales rendent compte. Alors que les hommes se protégeaient de l'hiver dans les premières représentations en restant chez eux, ils s'affairent ensuite à l'extérieur. Cette transition témoigne d'un nouveau rapport à la nature que les artistes participent à transmettre.

Le rôle de l'Église est prépondérant. Parce que les livres d'heures accompagnent le laïc dans ses pratiques liturgiques, l'image est choisie. C'est une façon pour l'Église d'asseoir son pouvoir: "Maîtriser le temps et être maître du calendrier qui permet de le maîtriser, c'est un instrument considérable du pouvoir [...] » (Le Goff, 2002). Malgré le caractère profane de certaines scènes, il est frappant d'ailleurs d'observer le nombre d'églises présentes en arrière-plan des enluminures hivernales.

Toute représentation paysagère s'inscrit donc dans un contexte qui lui est propre. Elle est destinée à un certain public qui conditionne des choix d'éléments du paysage. Ainsi, les représentations de l'hiver sont bien différentes selon les lieux et les époques, quoique marquées par des conditions météorologiques similaires. Les Hollandais mettront l'accent sur le patinage, les impressionnistes sur les chutes de neige et les hivers urbains... Les enluminures entre Moyen Âge et Renaissance ont elles aussi leur imagerie propre. Elles rappellent à quel point la vie du laïc était gouvernée par la religion, «par tous les temps ». 
L'auteur tient à remercier très chaleureusement Emmanuel Le Roy Ladurie, Danièle AlexandreBidon et Perrine Mane pour leurs précieux conseils, ainsi que Martine Tabeaud pour ses relectures attentives.

\section{BIBLIOGRAPHIE}

Alexandre, P., Le climat au Moyen Âge en Belgique et dans les régions voisines, Rhénanie, Nord de la France : recherches critiques d'après les sources narratives et essai d'interprétation, Louvain, Centre belge d'histoire rurale, 1976, $130 \mathrm{p}$.

Alexandre, P., Le Climat en Europe au Moyen Âge, Paris, École des hautes études en sciences sociales, 1987, $827 \mathrm{p}$.

Alexandre-Bidon, D., « Les jeux et sports d'hiver au Moyen Âge et à la Renaissance ", dans Jeux, sports et divertissements au Moyen Âge et à l'âge classique, Chambéry, Éditions du CTHS, 1993, p. $142-156$.

Baridon, M., Naissance et Renaissance du paysage, Arles, Actes Sud, 2006, 413 p.

Berque, A., Médiance, Paris, Belin, 2000, 157 p.

Cazelles, R., Les Très Riches Heures du Duc de Berry, préface de Umberto Eco, Lausanne/Paris, Édition Panthéon, 1988, 239 p.

Couleurs de neige, préface de G. Durand et introduction de G. Collot, Chambéry et Genève Albert Skira, 1992, exposition 17 janvier-19 mars 1992 au musée Savoisien de Chambéry.

Clark, K., L’Art du paysage, Paris, Gérard Monfort, 2006, 194 p.

Dehon, P.-J., Hiems Nascens. Premières représentations de l'hiver chez les poètes latins de la République, Rome, Editions dell' Ateneo, 2002, 93 p.

Duby, G., L’Art et la société. Le temps des cathédrales (980-1420), Paris, Gallimard, 1993, 379 p.

Ducos, J., Le Temps qu'il fait au Moyen Âge : phénomènes atmosphériques dans la littérature, la pensée scientifique et religieuse, Paris, Presses de l'université Paris-Sorbonne, 1988, 288 p.

Joutard, P., L'Invention du mont Blanc, Paris, Gallimard/Julliard, 1986.

Henish, B. A., The medieval Calendar Year, University Park, Pennsylvania state University Press, 1999, $232 \mathrm{p}$.

La Soudière, M. de, L'Hiver. À la recherche d'une morte-saison, Lyon, La Manufacture, 1987, 267 p.

Le Goff, J., Lefort, J. et Mane, P. (sous la dir. de), Les Calendriers : leurs enjeux dans l'espace et dans le temps, Paris, Somogy, 2002, 396 p., colloque de Cerisy du 1er au 8 juillet 2000.

Le Goff, J., « Bulletin météorologique au XIII ${ }^{\mathrm{e}}$ siècle », dans Mornet, E. et Morenzoni, F., Milieux naturels et espaces sociaux. Études offertes à Robert Delors, Paris, Publications de la Sorbonne, 1997, $761 \mathrm{p}$.

Le Roy Ladurie, E., Histoire humaine et comparée du climat, Paris, Fayard, 2004, vol. 1, 739 p. 
Luginbühl, Y., « Représentations du paysage, représentations de la société. Une lecture historique ", dans Jollivet, M. et Eizner, D. (sous la dir. de), L'Europe et ses campagnes, Paris, Fondation nationale des sciences politiques, 1996, $399 \mathrm{p}$.

Mane, P., Les Campagnes au Moyen Âge à travers les calendriers, Paris, La Martinière, 2004, 209 p.

Meiss, M., French Paintings in the Time of Jean de Berry. The Limbourg and This Contemporaries, Londres, Pierpont Morgan Library, 1974, 533 p. (texte) et environ 180 p. (figures), 2 vol.

Metzger, A., Plaisirs de Glace. Essai sur la peinture hollandaise hivernale du siècle d'or, Paris, Hermann, 2012, $120 \mathrm{p}$.

Muchembled, R., Culture populaire et culture des élites dans la France moderne, Paris, 1978, 398 p.

Pächt, O., Le paysage dans l'art italien : les premières études d'après nature dans l'art italien et les premiers paysages de calendrier, Paris, Gérard Montfort, 1991, 128 p.

Pächt, O., L'Enluminure médiévale, $1^{\text {re }}$ édition en langue originale en 1984, Paris, Éditions Macula, 1997, $224 \mathrm{p}$.

Pearsall, D. et Salter, E., Landscapes and Seasons of the Medieval World, Londres, P. Elek, 1973, 252 p.

Poirion, D. et Thomasset, C., L'Art de vivre au Moyen Âge, Paris, Éditions du Félin, 1995, 327 p.

Reichler, C., La Découverte des Alpes et la question du paysage, Génève, Georg, 2002, 256 p.

Vigneron, F., Les Saisons dans la poésie française des XIV et XV siècles, Paris, Honoré Champion, 2002, $650 \mathrm{p}$.

Van Straaten, E., Koud tot op het bot. De verbeelding van de winter in de zestiende en zeventiende eeuw in de Nederlanden, Gravenhague, Staatsuitgeverij's, 1977, 140 p.

Vasselin, M., «La naissance du paysage comme forme artistique dans l'Europe du XVI ${ }^{\mathrm{e}}$ siècle », Les paysages à l'époque moderne, association des historiens modernistes des universités françaises, Paris, Presses de l'université de Paris-Sorbonne, 2007, 115 p.

\section{NOTES}

1. Le retable des Portinari d'Hugo Van der Goes (vers 1475), exposé au musée des Offices à Florence, présente une scène d'adoration des bergers. Certes, le paysage est hivernal - les arbres sont dénudés, le ciel gris et la terre ocre - mais la neige est absente du paysage. Les scènes de nativité sont également dans leur très grande majorité privées de neige, voire printanières (comme dans le Bréviaire de Philippe le Bon, décoré par Jean le Tavernier vers 1460-1462).

2. La première représente une chute de neige dans la cours d'un château. Dans l'autre miniature, le paysage disparaît sous un manteau neigeux. Elle illustre la nouvelle 77 du Décameron de Boccace : Régnier va patienter tout un hiver au pied de la fenêtre d'Hélène. Les traces de pas dans la neige, tournant en rond, rappellent cette attente. Il sera vengé, l'été, en laissant Hélène au sommet d'une tourelle, brûlée par les rayons du soleil. 


\section{RÉSUMÉS}

L'objet de cet article est d'étudier comment se construit peu à peu un paysage climatique hivernal et quelles caractéristiques de l'hiver sont mises en avant. Absents des enluminures pendant une grande période, les paysages d'hiver vont être de plus en plus représentés à la fin du Moyen Âge dans les miniatures flamandes. Mais ce n'est pas un paysage réaliste que l'homme médiéval a sous ses yeux : le paysage est souvent choisi ou idéalisé. Les miniatures ne montrent qu'un des aspects du temps et des conditions de vie en hiver.

The subject of this article consists of studying how a climatic winter landscape is being progressively made and what features of winter are put forward. Absent from the illuminations for a great period, the winter landscapes are more and more represented at the end of the Middle Ages in the Flemish miniatures. But It is not a realistic landscape that the medieval man sees: the landscape is often chosen or idealised. The miniatures show only one side of the weather and life conditions in winter.

\section{INDEX}

Mots-clés : hiver, petit âge glaciaire, enluminures, représentations, paysage Keywords : winter, Little Ice Age, Illuminations, Representations, Landscape

\section{AUTEUR}

\section{ALEXIS METZGER}

Alexis Metzger est doctorant en géographie à l'université Paris-1 Panthéon-Sorbonne. Étudiant les mémoires et les représentations de la neige, il est rattaché au laboratoire ENEC UMR 8185 CNRS. Sa recherche est financée par la région île-de-France dans le cadre d'un contrat doctoral. alexis.metzger[at]yahoo[dot]fr 